\title{
Chemical potential of a two-component liquid in porous media: The case of unsaturated soil
}

\author{
J.C. Bénet ，F. J amin, M.S. El Youssoufi \\ Laboratoire de Mécanique et Génie Civil (LMGC), Université de Montpellier, CNRS, Montpellier, France
}

The aim of this paper is to propose a method to determine the chemical potential of two miscible components of a solution in porous media, particularly in the case of an unsaturated soil. It is limited to the capillary state; the equilibrium of the liquid phase in the soil is determined by gas-liquid interfaces whose geometry obeys Laplace's law. Deduction of the chemical potential of the two components from the chemical potential of pure water is based on the assumption that, for the same volume occupied by the fluid phase in the porous medium, the geometry of the fluid phase is independent of the composition of the solution.

The expressions of the chemical potentials of the two components were established. They show the superposition of two effects: the effect of interfacial forces and the effect of the presence of the other component. A study of the water-alcohol solution in a clayey silty sand soil was then conducted to alcohol mole fractions between 0 and 0.15 and water content in the 5\%-15\% range. In this field, the chemical potential of the water was seen to be affected by the capillary effects below a water content of $10 \%$. Above that, its variation as a function of the mole fraction of alcohol differed little from that of the potential of the water in a free solution. The chemical potential of the alcohol was little affected by the capillary effects.

Keywords:

Chemical potential; Soil water retention curve; Porous media; Unsaturated soil

\section{Introduction}

Thermodynamic modelling of unsaturated soils in areas such as environmental geomechanics, agronomy, etc., implies knowledge of the state of the various components of the liquid phase. In soil, the liquid phase may be multi-

\footnotetext{
* Corresponding author.

E-mail address: jean-claude.benet@umontpellier.fr (J.C. Bénet).
}

component, especially in polluted areas. Remediation by venting requires continuous treatment for several months or even years, at low extraction rates. ${ }^{1}$ Several authors (Poulsen et al. ${ }^{2}$, Rathfelder et al. $^{3}$ ) stress the marked influence of the NAPL phase-gas phase change kinetics on decontamination. The effect of air velocity and air pollutant content has been analysed. ${ }^{4}$ It is clear from these studies that ventilation decontamination rate is largely determined by the difference between the chemical potential of the components in liquid and gas phase. ${ }^{5,6}$ 
The introduction of the chemical potentials of the various components makes it possible to describe the thermodynamic equilibrium and transfers in porous media. ${ }^{7}$ This thermodynamic function is defined as the derivative of the internal energy with respect to the mass change of a component with entropy, volume and mass of other component constants. ${ }^{8,9}$ In a reversible evolution, it represents the mechanical work to be done (or received) when extracting (or introducing) a unit mass of the component in the system. This quantity is therefore representative of the action of the rest of the system on the component.

In a previous publication, ${ }^{10}$ it was shown that the experimental techniques available, i.e. tensiometry and the use of saturated salt solutions, make it possible to establish the relationship between chemical potential and water content when the liquid phase of the porous medium is composed of pure water. This relationship has been established in the isothermal case for media having various structures: soils, gels and food products. We intend to extend this result to the case where the pore space contains a binary solution of two immiscible liquids and a gas phase. In this case measuring the pressure of the solution is not sufficient to characterize the thermodynamic state of the two components.

The aim of this paper is to propose a method to determine the chemical potential of the components of a solution of two miscible liquids in porous media, particularly in the case of unsaturated soil. It is limited to the capillary state; the equilibrium of the liquid phase in the soil is determined by gas/liquid interfaces whose geometry obeys Laplace's law. The water-alcohol solution will be considered as a case study.

\section{Chemical potential of a single liquid in porous media}

\subsection{Measurement method}

Measurement of chemical potential is based on its fundamental property: if in a system $\alpha$, a component $k$ is in thermodynamic equilibrium with the same component in a system $\beta$, through a membrane that is permeable to component $k$ only, the second principle of thermodynamics implies ${ }^{11}$ :

$\mu_{\alpha k}=\mu_{\beta k}$

where $\mu_{\alpha k}$ and $\mu_{\beta k}(\mathrm{~J} / \mathrm{kg})$ are the chemical potentials of component $k$ in the two systems.

In the case of a single liquid in a porous medium, three different circumstances may be distinguished related to different configurations of the liquid with respect to the solid in the porous medium. The first concerns the circumstances where gas-liquid meniscus equilibrium is governed by Laplace's law, ${ }^{8}$ the liquid phase being continuous. This configuration is sometimes called funicular. The surface tension at the liquid-gas interface creates a pressure difference between the gas and the liquid:

$p_{g}-p_{\ell}=\sigma\left(\frac{1}{R_{1}}+\frac{1}{R_{2}}\right)$

where $p_{g}$ and $p_{\ell}$ are the pressures of the gas and liquid phases, $\sigma$ is the surface tension of the liquid, and $R_{1}$ and
$R_{2}$ are the principal radii of curvature of the liquid-gas interfaces. Gagneux and Millet ${ }^{12}$ give extensive details on different configurations of this curvature. In Eq. (2), the radii are considered as positive when the curvature is turned into the interior of the gas phase.

Relationship (2) determines the geometry of the interface. In order to measure the chemical potential in the liquid in this case, the semi permeable membrane used is a high air entry disk (HAED) which is impermeable to air but not to liquid. The chemical potential of liquid in this configuration is evaluated by measuring the pressure of the single liquid $p_{\ell}$ (whose specific density is $\rho_{\ell}^{*}$ ) on the other side of the HAED:

$\mu_{\ell}=\mu_{\ell}^{0}\left(T, p_{a t m}\right)+\frac{p_{\ell}}{\rho_{\ell}^{*}}$

where $\mu_{\ell}^{0}\left(T, p_{a t m}\right)$ is a constant that depends on temperature and a reference pressure. The latter is taken equal to the atmospheric pressure $p_{a t m}$; the result is that $p_{\ell}$ is the effective pressure of the liquid phase and $p_{g}$ is the effective pressure of the gas phase equal to $0 \mathrm{kPa}$.

In the case of water, the measurement range of tensiometry is restricted, first, by the difficulty of ensuring perfect continuity of the phase of water through the porous plate and, second, by the possibility of the water cavitation occurring at low pressure. Therefore, to widen the measurement range towards higher values of chemical potential, an over-pressure can be applied in the gasphase of the medium. ${ }^{13}$ This technique regularly leads to experimental results within a wide pressure range from $1 \times 10^{2}$ to $5 \times 10^{5} \mathrm{~Pa}$ (Leong and Rahardjo ${ }^{14}$, Salager et al. ${ }^{13}$ ). However, liquid relative pressure values below $-10^{5} \mathrm{~Pa}$ should be carefully interpreted. ${ }^{15}$

The second circumstance concerns the state of the liquid when it is adsorbed on the solid surface. This configuration is often called hygroscopic. This state is characterized by a substantial deviation of the liquid vapour pressure from its saturation vapour pressure. A boundary layer, between the porous medium and the atmosphere, allows to pass the water molecules and so it plays the role of a semi-permeable membrane for water, allowing to apply the relation (1). Hence the chemical potential is estimated from the relative humidity HR of the vapour in equilibrium, with the relationship:

$\mu_{\ell}=\mu_{\ell}^{0}\left(T, p_{a t m}\right)+\frac{R T}{M_{\ell}} \ln (H R)$

where $R$ is the gas constant and $M_{\ell}$ is the molar mass of the liquid. The reference state corresponds to pure water at a flat surface, at atmospheric pressure and in equilibrium with its vapour at temperature $T$; the reference chemical potentials in (3) and (4) are identical. Thereafter the chemical potential reference is taken as zero.

Between funicular and hygroscopic state, there is a set of intermediate configurations that includes the presence of thin film surface layers and gas-liquid inter-granular meniscus sometimes called pendular. HAED method is not efficient enough to characterize the relationship between the pressure in water in the porous medium and water in the tensiometer tank. That results from the fact that the meniscus radii in porous stone are too large to 


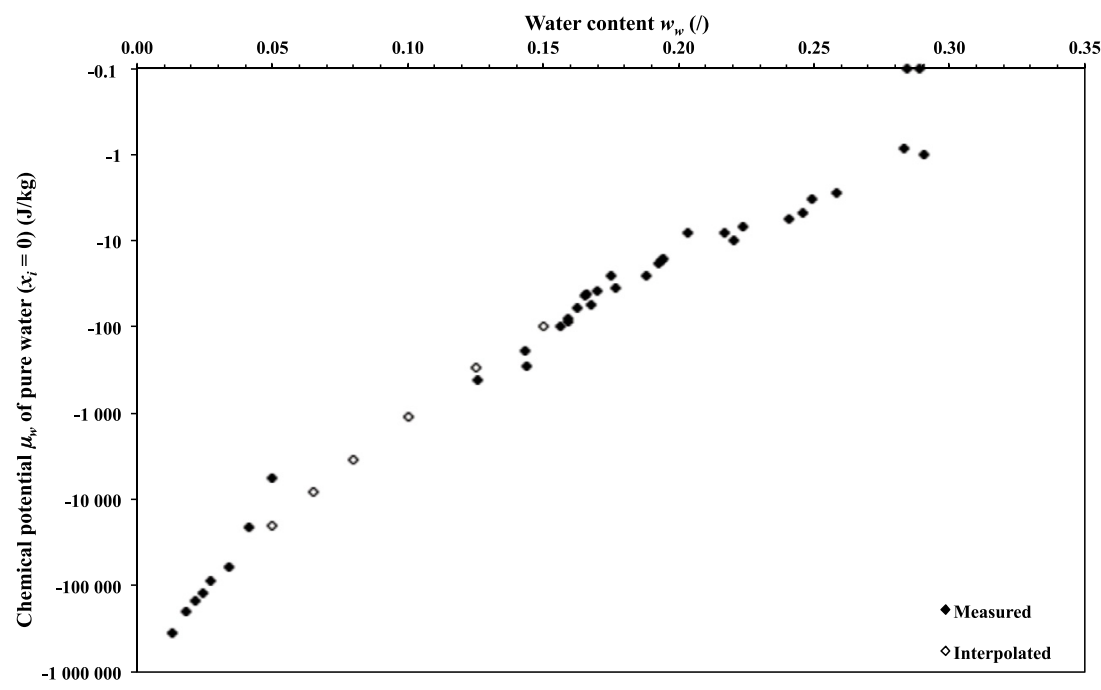

Fig. 1. Variation of the chemical potential of water as a function of water content for clayey silty sand for $T=20{ }^{\circ} \mathrm{C}$.

significantly lower the vapour activity below 1 . For such a configuration, the two previous measurement methods are inadequate. However, the transition from hygroscopic field to funicular field is not abrupt; in certain range of the liquid content, there are bridges with a capillary configuration, for which it is possible to define a difference of pressure across the interface. Eq. (1) must be satisfied for the component in all its forms: capillary bridges, liquid films on the surface of the solid and liquid vapour. The thermodynamic state of the fluid can be characterized by a single chemical potential. The interpolation steps between the funicular and hygroscopic areas are generally easier for a variety of structures: powders, gels, food products. ${ }^{10}$ This interpolation leads to establishing the relationship between the chemical potential and water content for the entire range of water content of the medium.

\subsection{Relationship between chemical potential and pure water content in soil}

The porous media used in the experimental study that follows is clayey silty sand, comprising $40 \%$ quartz, $50 \%$ calcite and $10 \%$ clay, ${ }^{16,13}$ with particle size less than $2 \mathrm{~mm}$. During testing, it was compacted to achieve a bulk density of the solid phase of $1500 \mathrm{~kg} / \mathrm{m}^{3}$, corresponding to a porosity of 0.43 .

In the funicular configuration, the chemical potential of pure water is measured using the suction plate. ${ }^{16,13}$ The initial state is the saturated state. The temperature is set at $20^{\circ} \mathrm{C}$. The suction plate gives the relationship between the pressure of the water in the soil and the water content. It is possible to apply gas pressure up to $500 \mathrm{kPa}$ using this method to establish the relationship in the range $[1 \mathrm{kPa}$; $500 \mathrm{kPa}]$. The chemical potential of the water in the soil is evaluated using ( 3 ).

In the hygroscopic area, the chemical potential of pure water is measured with the saturated salt solution method. ${ }^{16}$ The initial state is the saturated state. The temperature is set at $20^{\circ} \mathrm{C}$. The equilibrium between water in the soil and water in salt solutions makes it possible to calculate the chemical potential of water in the soil by using the relation (4).

Fig. 1 shows the variation in logarithmic scale of the chemical potential of water in the soil as a function of water content. It highlights the interpolated part of the curve. Measurements using liquid vapour equilibrium could be made from a water content of $5 \%$, which corresponds (Fig. 1) to a chemical potential of $5000 \mathrm{~J} / \mathrm{kg}$. Eq. (4) gives an equilibrium relative humidity of $96.3 \%$, which shows that adsorption is weak. So we are still in a state managed by capillary effects, although imperfect porous stone does not allow measurement up to this value. Subsequently, the capillary fields also dominate the funicular area and the pendulum field; in both cases, the capillary determines the chemical potential of the fluid. The study will be focused at the intermediate area of water content between $5 \%$ and $15 \%$.

\section{Chemical potential of a solution of two miscible liquids in porous media}

\subsection{System definition}

We examine the case of a liquid phase consisting of pure water and a water-miscible liquid (e.g. water-alcohol, water-polyethylene glycol, etc.), referred to hereinafter as component $i$. The considered medium is composed of an isotropic porous non-deformable skeleton whose voids are filled by the binary liquid solution and a gas phase. The temperature is assumed to be uniform and constant. The gas phase consists of air and the vapour of the two liquids in equilibrium with the liquid solution. The total pressure of the gas phase is assumed to be uniform and constant and equal to the atmospheric pressure; the partial air pressure varies to meet this assumption. The liquid phase is assumed to be incompressible at the pore scale. The system considered thereafter is the matter in a volume containing the unit mass of solid phase $\left(m_{s}=1 \mathrm{~kg}\right)$. This 
volume $V$ is given by:

$V=\frac{1}{\rho_{s}}$

where $\rho_{s}$ is the apparent density $\left(\mathrm{kg} / \mathrm{m}^{3}\right)$.

So, the masses of water and component $i$ (respectively denoted as $m_{w}$ and $m_{i}$ ) in the system are equal to the water content and component $i$ content defined respectively by $w_{w}=m_{w} / m_{s}$ and $w_{i}=m_{i} / m_{s}$. The chemical potential of water and component $i$ is defined using the free energy potential $\tilde{f}\left(m_{w}, m_{i}\right)^{11}$ :

$\mu_{w}=\left(\frac{\partial \tilde{f}}{\partial m_{w}}\right)_{m_{i}}=\left(\frac{\partial \tilde{f}}{\partial w_{w}}\right)_{w_{i}}$

$\mu_{i}=\left(\frac{\partial \tilde{f}}{\partial m_{i}}\right)_{m_{w}}=\left(\frac{\partial \tilde{f}}{\partial w_{i}}\right)_{w_{w}}$.

The derivatives of the chemical potentials need to satisfy the condition (8):

$$
\left(\frac{\partial \mu_{w}}{\partial w_{i}}\right)_{w_{w}}=\left(\frac{\partial \mu_{i}}{\partial w_{w}}\right)_{w_{i}} .
$$

The number of moles per unit mass for the two components is given by:

$n_{w}=\frac{w_{w}}{M_{w}}$

$n_{i}=\frac{w_{i}}{M_{i}}$,

where $M_{w}$ and $M_{i}$ are respectively the molar mass of the pure water and the component $i$.

The molar fractions are given by:

$$
\begin{aligned}
& x_{w}=\frac{n_{w}}{n_{w}+n_{i}} \\
& x_{i}=\frac{n_{i}}{n_{w}+n_{i}} .
\end{aligned}
$$

With:

$x_{w}=1-x_{i}$.

According to (10)-(12), variable $x_{i}$ depends on the two state variables:

$w_{i}=\frac{M_{i}}{M_{w}} \frac{x_{i}}{\left(1-x_{i}\right)} w_{w}$.

With relationships used later:

$$
\begin{aligned}
& \left(\frac{\partial x_{i}}{\partial w_{i}}\right)_{w_{w}}=-\left(\frac{\partial x_{w}}{\partial w_{i}}\right)_{w_{w}}=\frac{M_{w}}{M_{i}} \frac{\left(1-x_{i}\right)^{2}}{w_{w}} \\
& \left(\frac{\partial x_{i}}{\partial w_{w}}\right)_{w_{i}}=-\left(\frac{\partial x_{w}}{\partial w_{w}}\right)_{w_{i}}=-\frac{M_{i}}{M_{w}} \frac{x_{i}^{2}}{w_{i}}=\frac{x_{i}\left(1-x_{i}\right)}{w_{w}} .
\end{aligned}
$$

$V_{\ell}^{*}$ denotes the volume occupied by the liquid phase in the medium at the pore scale, $\rho_{w}^{*}$ and $\rho_{i}^{*}$ the specific densities of water and component $i$. Assuming the additivity of volumes:

$V_{\ell}^{*}=\frac{w_{w}}{\rho_{w}^{*}}+\frac{w_{i}}{\rho_{i}^{*}}$.

Using (14), this relation can be written:

$V_{\ell}^{*}=\frac{w_{w}}{\rho_{w}^{*}}\left(1+\frac{M_{i}}{M_{w}} \frac{x_{i}}{\left(1-x_{i}\right)} \frac{\rho_{w}^{*}}{\rho_{i}^{*}}\right)$

$V_{\ell}^{*}=\frac{w_{i}}{\rho_{w}^{*}}\left(\frac{\rho_{w}^{*}}{\rho_{i}^{*}}+\frac{M_{w}}{M_{i}} \frac{x_{w}}{\left(1-x_{w}\right)}\right)$.

In case the pure water saturates the medium $x_{i}=0$, the water content is denoted as $w_{w}^{\text {sat }}$, (18) gives the void volume $V_{v}$ of the porous medium:

$V_{v}=\frac{w_{w}^{\text {sat }}}{\rho_{w}^{*}}$.

For a given value of $w_{w}$, the fraction $x_{i}$ cannot grow indefinitely $\left(V_{\ell}^{*} \leq V_{v}\right)$; it must meet the following condition:

$\frac{x_{i}}{\left(1-x_{i}\right)} \leq \frac{M_{w}}{M_{i}} \frac{\rho_{i}^{*}}{\rho_{w}^{*}} \frac{\left(w_{w}^{\text {sat }}-w_{w}\right)}{w_{w}}=A$.

The limit $x_{i}^{\lim }$ value of the fraction $x_{i}$ is given by the equation:

$x_{i}^{\lim }=\frac{A}{1+A}$.

3.2. Expression of the chemical potential of water and component $i$

To assess the mass chemical potential of water in the solution of a porous medium, we imagined the experiment shown diagrammatically in Fig. 2.

The porous medium containing a binary solution of water and a component $i$ in $(A)$ is in communication, through a porous stone, with compartment (B) containing the only solution. It ensures equality between the pressure $p_{\ell}^{\mathrm{B}}$ in $\mathrm{B}$ and the pressure of the liquid phase $p_{\ell}^{\mathrm{A}}$ in the pores of $A$ :

$p_{\ell}^{\mathrm{A}}=p_{\ell}^{\mathrm{B}}$.

Firstly, consider an ideal semi-permeable membrane to water separated compartments B and C; C contains pure water and we denote $p_{\ell}^{C}=p_{w}$ as the pressure of pure water in $C$. The pressure difference between these compartments is equal to the osmotic pressure $\Pi_{w}$ :

$\Pi_{w}=p_{\ell}^{\mathrm{B}}-p_{w}$.

Thereafter the gas phase is at atmospheric pressure; the pressure of the liquid phase used in relations (23) and (24) is the effective pressure. At equilibrium, the chemical potential of the water component is the same in A, B, C. It is measured in C; using (3):

$\mu_{w}\left(w_{w}, x_{i}\right)=\frac{p_{w}}{\rho_{w}^{*}}=\frac{p_{\ell}^{\mathrm{B}}-\Pi_{w}}{\rho_{w}^{*}}$. 


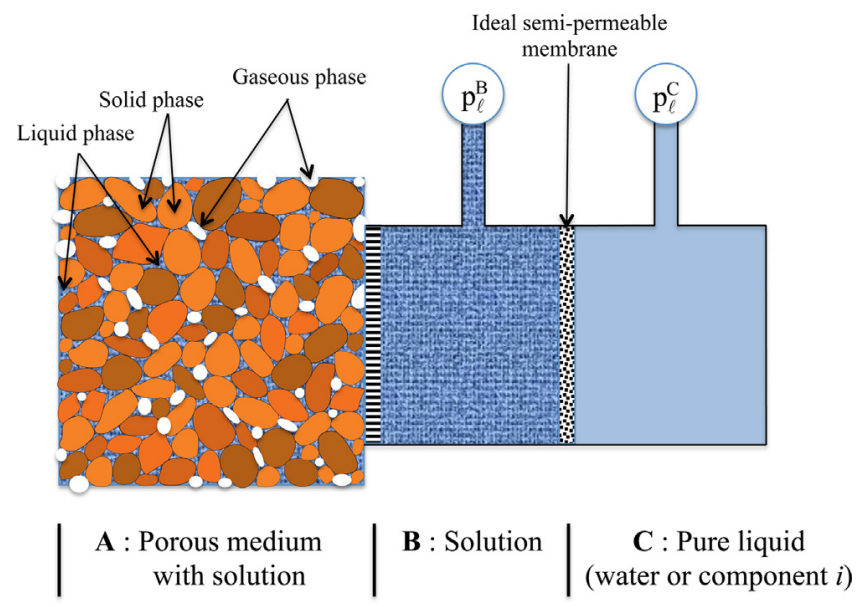

Fig. 2. Experimental device to evaluate the chemical potential in the solution of a capillary porous media.

Measuring pure water pressure in compartment $C$ gives the chemical potential of the water component in the solution of the porous medium (A). If we know the partial pressure of the vapour in equilibrium with the water $p_{v w}\left(x_{i}\right)$ in compartment $\mathrm{B}$ and $p_{v w}^{0}=p_{v w}\left(x_{i}=0\right)$ in compartment $\mathrm{C}$, neglecting fluid compressibility osmotic pressure is given by ${ }^{8}$ :

$\Pi_{w}=\rho_{w}^{*} \frac{R T}{M_{w}} \ln \left(\frac{p_{v w}^{0}}{p_{v w}\left(x_{i}\right)}\right)$

where $M_{w}$ is the molar mass of water. From a thermodynamics point of view, there is no difference between a brine solution (salt dissolved in the water) and a solution of two miscible liquids. Thus, the relation (26) is valid in both cases. According to Guggenheim, ${ }^{8}$ the equilibrium pressure $p_{v w}\left(x_{i}\right)$ depends little on the outside pressure. The chemical potential of water in the medium (25) is put in the form:

$\mu_{w}\left(w_{w}, x_{i}\right)=\frac{p_{\ell}^{\mathrm{B}}\left(w_{w}, x_{i}\right)}{\rho_{w}^{*}}+\frac{R T}{M_{w}} \ln \left(\frac{p_{v w}\left(x_{i}\right)}{p_{v w}^{0}}\right)$.

Using Laplace's law (2):

$p_{\ell}^{\mathrm{B}}\left(w_{w}, x_{i}\right)=-\sigma\left(x_{i}\right)\left(\frac{1}{R_{1}}+\frac{1}{R_{2}}\right)$

where $\sigma\left(x_{i}\right)$ is the surface tension of the solution.

We consider two states: the first (state 1 ) corresponding to pure water $x_{i}=0$ occupying a volume $V_{\ell}^{*}$ in the volume $V$, and the second (state 2) $x_{i} \neq 0$ corresponding to a solution, occupying the same volume $V_{\ell}^{*}$. Assuming that the liquid phase is incompressible, and that the solid phase is perfectly wetting for water and for the binary solution and the surface tension is uniform, the geometry of the liquid phase in the medium, as determined by capillary effects, is the same in both cases. The principal radii of curvature $R_{1}$ and $R_{2}$ of the menisci are independent of $x_{i}$, which leads, using (28), to:

$\frac{p_{\ell}^{\mathrm{B}}\left(w_{w}, x_{i}\right)}{p_{\ell}^{\mathrm{B}}\left(w_{w}^{0}, x_{i}=0\right)}=\frac{\sigma\left(x_{i}\right)}{\sigma\left(x_{i}=0\right)}=f\left(x_{i}\right)$ where $w_{w}^{0}$ is the water content for state 1 . According to (18), equal volumes in the states 1 and 2 are reflected by a relationship between $w_{w}^{0}$ and $w_{w}$ :

$w_{w}^{0}=w_{w}\left(1+\frac{M_{i}}{M_{w}} \frac{x_{i}}{\left(1-x_{i}\right)} \frac{\rho_{w}^{*}}{\rho_{i}^{*}}\right)$.

For pure water, the chemical potential is given, using (3), by:

$\mu_{w}\left(w_{w}^{0}, x_{i}=0\right)=\frac{p_{\ell}^{\mathrm{B}}\left(w_{w}^{0}, x_{i}=0\right)}{\rho_{w}^{*}}$.

With (29) and (31), the chemical potential of water in the solution of porous media (27) is:

$$
\begin{aligned}
\mu_{w}\left(w_{w}, x_{i}\right)= & \mu_{w}\left(w_{w}^{0}, x_{i}=0\right) f\left(x_{i}\right) \\
& +\frac{R T}{M_{w}} \ln \left(\frac{p_{v w}\left(x_{i}\right)}{p_{v w}^{0}}\right) .
\end{aligned}
$$

Secondly, consider an ideal semi-permeable membrane to component $i$ separated compartments B and C; C contains the pure component $i$ and we denote $p_{\ell}^{C}=p_{i}$ as the pressure of pure component $i$ in $C$. As pure water case, all Eqs. (24)-(26) are identically applied to the component $i$ by replacing $w$ by $i$. Following the reasoning that leads to (27), the chemical potential of $i$ in the three compartments is given by:

$\mu_{i}\left(w_{i}, x_{w}\right)=\frac{p_{\ell}^{\mathrm{B}}\left(w_{i}, x_{w}\right)}{\rho_{i}^{*}}+\frac{R T}{M_{i}} \ln \left(\frac{p_{v i}\left(x_{w}\right)}{p_{v i}^{0}}\right)$

where $p_{v i}^{0}=p_{v i}\left(x_{w}=0\right)$ is the equilibrium vapour pressure of pure component $i$ in compartment $C$.

The relation analogous to (29) is written:

$\frac{p_{\ell}^{\mathrm{B}}\left(w_{i}, x_{w}\right)}{p_{\ell}^{\mathrm{B}}\left(w_{i}^{0}, x_{w}=0\right)}=\frac{\sigma\left(x_{w}\right)}{\sigma\left(x_{w}=0\right)}=g\left(x_{w}\right)$

where $w_{i}^{0}$ corresponds to the pure liquid $i$, this value can be calculated by (19). 
Applying Eq. (29) in the special case of the two pure liquids:

$\frac{p_{\ell}^{\mathrm{B}}\left(w_{i}^{0}, x_{w}=0\right)}{p_{\ell}^{\mathrm{B}}\left(w_{w}^{0}, x_{i}=0\right)}=\frac{\sigma\left(x_{w}=0\right)}{\sigma\left(x_{i}=0\right)}=C$.

Molar fractions $x_{i}$ and $x_{w}$ are being linked by (13):

$g\left(x_{w}\right)=\frac{f\left(x_{i}\right)}{C}$.

For pure $i, \rho_{i}^{*}$ being the specific density of component $i$, the chemical potential of component $i$ is:

$\mu_{i}\left(w_{i}^{0}, x_{w}=0\right)=\frac{p_{\ell}^{\mathrm{B}}\left(w_{i}^{0}, x_{w}=0\right)}{\rho_{i}^{*}}$.

Introducing (31) and (37) in (35) gives:

$\frac{\rho_{i}^{*} \mu_{i}\left(w_{i}^{0}, x_{w}=0\right)}{\rho_{w}^{*} \mu_{w}\left(w_{w}^{0}, x_{i}=0\right)}=C$.

This relationship gives the variation of the chemical potential of pure $i$ in the porous medium as a function of the content of $i$, knowing the variation of the chemical potential of pure water with the water content. With (37), relation (33) becomes:

$$
\begin{aligned}
\mu_{i}\left(w_{i}, x_{w}\right)= & \mu_{i}\left(w_{i}^{0}, x_{w}=0\right) g\left(x_{w}\right) \\
& +\frac{R T}{M_{i}} \ln \left(\frac{p_{v i}\left(x_{w}\right)}{p_{v i}^{0}}\right) .
\end{aligned}
$$

According to (36) and (38), (39) can also be written in terms of the variable $x_{i}$ to locate $\mu_{i}$ and $p_{v i}$ :

$$
\begin{aligned}
\mu_{i}\left(w_{i}, x_{i}\right)= & \frac{\rho_{w}^{*}}{\rho_{i}^{*}} \mu_{w}\left(w_{w}^{0}, x_{i}=0\right) f\left(x_{i}\right) \\
& +\frac{R T}{M_{i}} \ln \left(\frac{p_{v i}\left(x_{i}\right)}{p_{v i}^{0}}\right) .
\end{aligned}
$$

Relations (32) and (40) show the actions of the interfaces (first term of the second member) and the mutual action of the fluid (second term of the second member) on the chemical potentials of the two fluids. The second derivative of the free energy is obtained from (33) using (15):

$$
\begin{aligned}
& \frac{\partial^{2} \tilde{f}}{\partial w_{i} \partial w_{w}}=\frac{\partial \mu_{w}}{\partial w_{i}}=\frac{\partial \mu_{w}}{\partial x_{i}} \frac{\partial x_{i}}{\partial w_{i}} \\
& =\frac{\left(1-x_{i}\right)^{2}}{w_{w}}\left[\frac{M_{w}}{M_{i}} \mu_{w}\left(w_{w}^{0}, x_{i}=0\right) \frac{\partial f\left(x_{i}\right)}{\partial x_{i}}\right. \\
& \left.\quad+\frac{R T}{M_{i}} \frac{\partial \ln \left(p_{v w}\left(x_{i}\right)\right)}{\partial x_{i}}\right] .
\end{aligned}
$$

It can also be deduced from (40) using (16)

$$
\begin{aligned}
& \frac{\partial^{2} \tilde{f}}{\partial w_{w} \partial w_{i}}=\frac{\partial \mu_{i}}{\partial w_{w}}=\frac{\partial \mu_{i}}{\partial x_{w}} \frac{\partial x_{w}}{\partial w_{w}}=\frac{x_{i}\left(1-x_{i}\right)}{w_{w}} \\
& \times\left[\mu_{i}\left(w_{i}^{0}, x_{w}=0\right) \frac{\partial g\left(x_{i}\right)}{\partial x_{i}}-\frac{R T}{M_{i}} \frac{\partial \ln \left(p_{v i}\left(x_{i}\right)\right)}{\partial x_{i}}\right] .
\end{aligned}
$$

According to (36), (38), (11) and (12), the ratio of the first two terms in (41) and (42) is equal to one:

$$
\begin{gathered}
\frac{\left(1-x_{i}\right)}{x_{i}} \frac{M_{w}}{M_{i}} \frac{\mu_{w}\left(w_{w}^{0}, x_{i}=0\right) \frac{\partial f\left(x_{i}\right)}{\partial x_{i}}}{\mu_{i}\left(w_{i}^{0}, x_{w}=0\right) \frac{\partial g\left(x_{i}\right)}{\partial x_{i}}} \\
=\frac{x_{w}}{x_{w}} \frac{M_{w}}{M_{w}} \frac{\rho_{i}^{*}}{\rho_{w}^{*}}=\frac{n_{w}}{n_{i}} \frac{M_{w}}{M_{i}} \frac{\rho_{i}^{*}}{\rho_{w}^{*}}=1 .
\end{gathered}
$$

Using Duhem-Margule relationship ${ }^{8}$ :

$$
\left(1-x_{i}\right) \frac{\partial \ln \left(p_{v w}\left(x_{i}\right)\right)}{\partial x_{i}}+x_{i} \frac{\partial \ln \left(p_{v i}\left(x_{i}\right)\right)}{\partial x_{i}}=0 .
$$

The expressions (41) and (42) are equivalent.

\section{Case of a water-alcohol solution in a clayey silty sand}

\subsection{Water-alcohol solution}

The molar mass of pure alcohol is $0.046 \mathrm{~kg} / \mathrm{mole}$ and its density is $789 \mathrm{~kg} / \mathrm{m}^{3}$. The study is restricted to water contents between $5 \%$ and $15 \%$ and alcohol mole fractions between 0 and 0.15 . In this area, satisfying the relation (22), the effects of the surface layers and mutual effects of the two fluids are in the same order. Variations in partial pressures $p_{v w}\left(x_{i}\right)$ and $p_{v i}\left(x_{i}\right)$ above a water-ethyl alcohol solution at $25{ }^{\circ} \mathrm{C}$ in the free state, are given in Fig. $3 .{ }^{8}$

The chemical potentials of water and alcohol in the free state (outside the porous medium) are derived from the relations (4) with, as in Section 3, zero constant for the pure fluids at atmospheric pressure. Fig. 4 shows the variation of the two chemical potentials in relation with $x_{i}$.

Fig. 5 shows the functions defined by (29) and (34) from the surface tension of a water-alcohol solution. ${ }^{8}$ For this solution the coefficient $C$ defined by (35) is equal to 0.328 .

\subsection{Chemical potential of water and alcohol in the clayey silty sand}

The water content adopted to set the curves is the water content $w_{w}^{0}$. In the water content range studied, $w_{w}^{0}$ given by (30), varies almost linearly, the ratio $w_{w}^{0} / w_{w}$ is one for $x_{i}=0$ and 1.57 , for $x_{i}=0.15$.

Fig. 6 shows the variation of the chemical potential of water as a function of $x_{i}$ for $w_{w}^{0}=6 \%$. This figure brings up the first term of (32) related to the effects of interfaces, and the second term in (32) related to the presence of alcohol. Increasing $x_{i}$ causes lowering of surface tension (Fig. 5). The difference between the pressure of the gas phase and the pressure of the liquid phase decreases. The pressure of the gas phase being constant (equal to the atmospheric pressure), this translates into an increase in the pressure of the liquid phase and therefore the increase of the first term of (32). For the second term of Eq. (32) there is a decrease due to the decrease of the partial pressure of water in equilibrium with the alcohol content (Fig. 4). For $x_{i}=0$, the interface effect occurs in accordance with the relationship shown in Fig. 1. When $x_{i}$ increases, the effect of the presence of alcohol gradually prevails over the interface effect. 


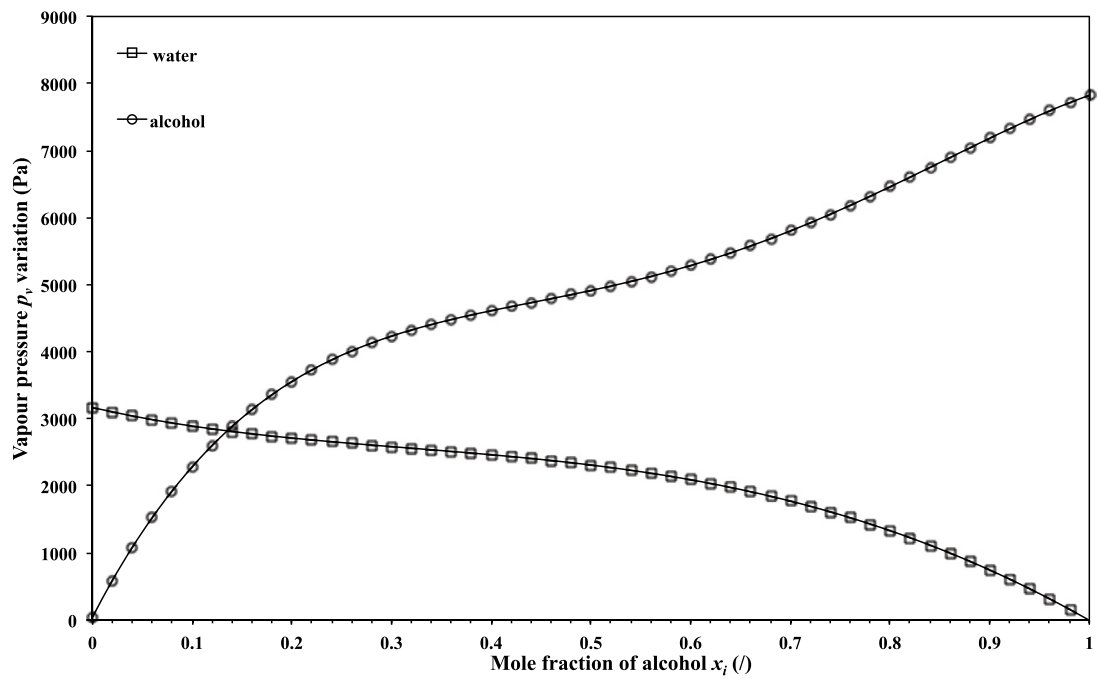

Fig. 3. Vapour pressure variation of the water and alcohol in a solution in relation to the mole fraction of alcohol solution.

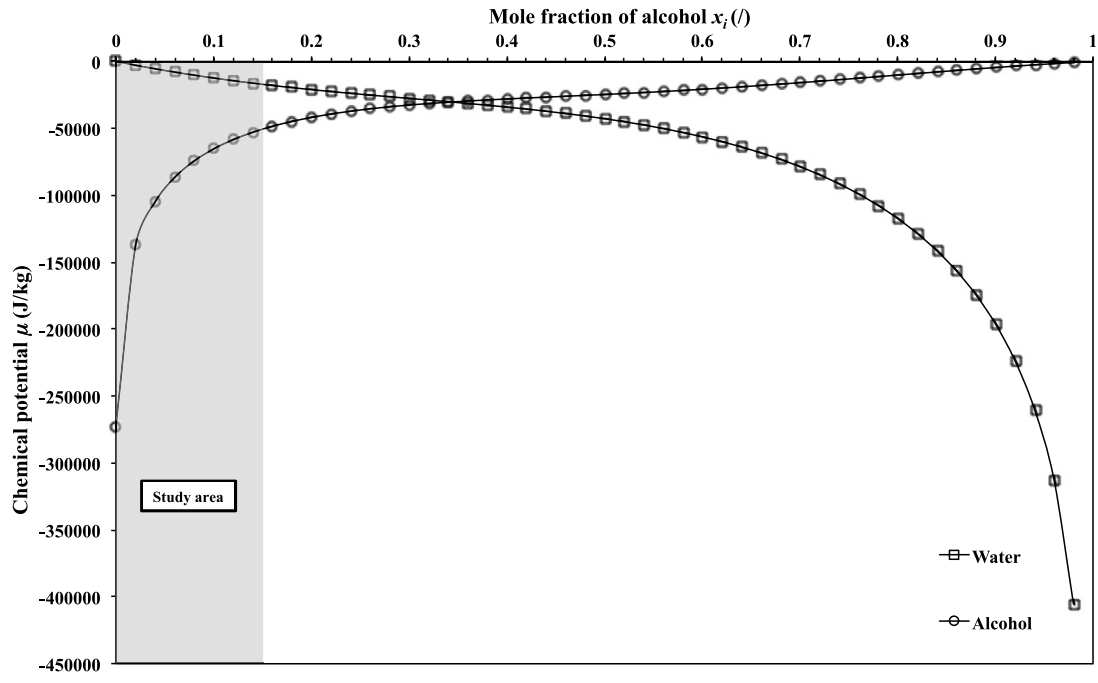

Fig. 4. Chemical potential of water and alcohol in a free state solution.

Fig. 7 shows the change in the chemical potential of water as a function of $x_{i}$ for various water contents $w_{w}^{0}$ of the field of study. The decrease of the chemical potential with $x_{i}$ is recognized for all water contents as indicated above. This figure shows the variation of the chemical potential of water for a free solution. This variation is consistent with Fig. 4 in the field of study. It can be seen that the distance between the curves increases as the water content decreases; this shows the increasing effect of the interfaces. When the water content exceeds $10 \%$, the effect weakens; the soil solution behaves like a water-alcohol solution in the free state.

Fig. 8 shows the variation of the chemical potential of water as a function of the water content for various values of $x_{i}$. The curve for $x_{i}=0$ conforms to the curve of Fig. 1, with the dotted portion corresponding to the hygroscopic field. The contribution of component $i$ tends to lower the chemical potential. This figure also shows that, for all $x_{i}$ the curves tend asymptotically to the curve representing the hygroscopic behaviour when $w_{w}^{0}$ tends to zero. This suggests that in the hygroscopic field, the effect of component $i$ in the chemical potential of water becomes progressively weaker as does the interface effect. This reflects the fact that liquid-solid bond strength increases significantly when water content tends to zero.

Fig. 9 shows the variation of the chemical potential of alcohol as a function of $x_{i}$ for various water contents. In the field explored, curves deviate slightly from the curve corresponding to a free solution. The interface effect (Fig. 1) is an order well below the alcohol concentration effect (Fig. 4); it begins to manifest water content of about $5 \%$.

\section{Conclusions}

The assumption behind the assessment of chemical potentials of the components of a binary solution in a porous medium from the chemical potential of pure water is that, for the same volume occupied by the fluid phase 


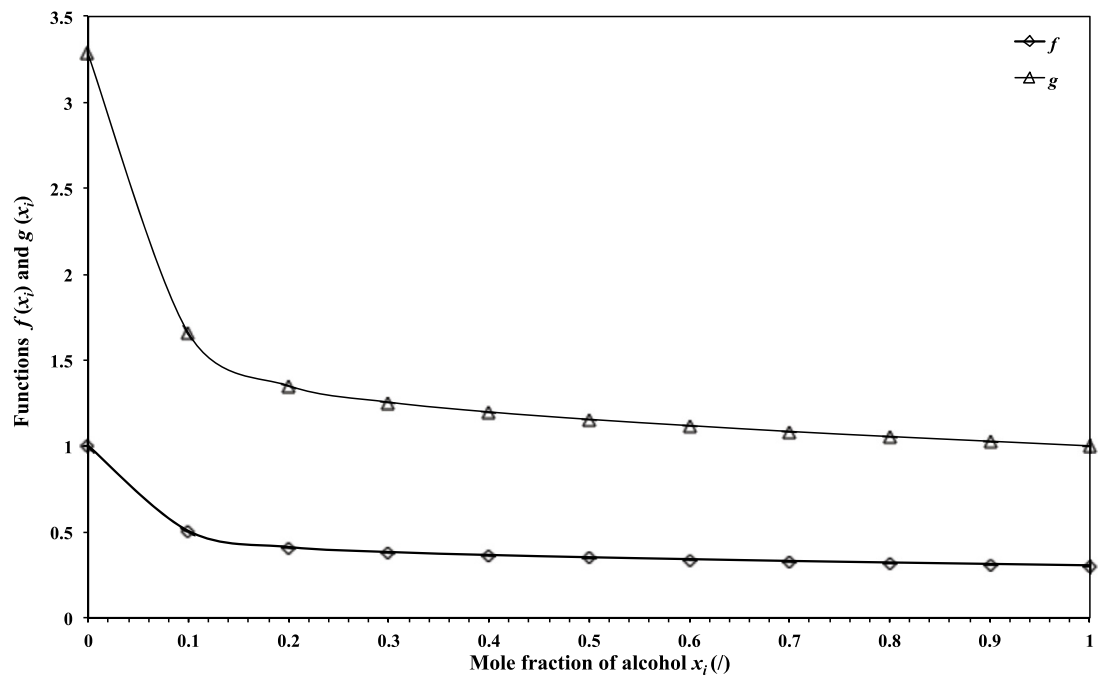

Fig. 5. Functions $f\left(x_{i}\right)$ and $g\left(x_{i}\right)$ for the water-alcohol solution.

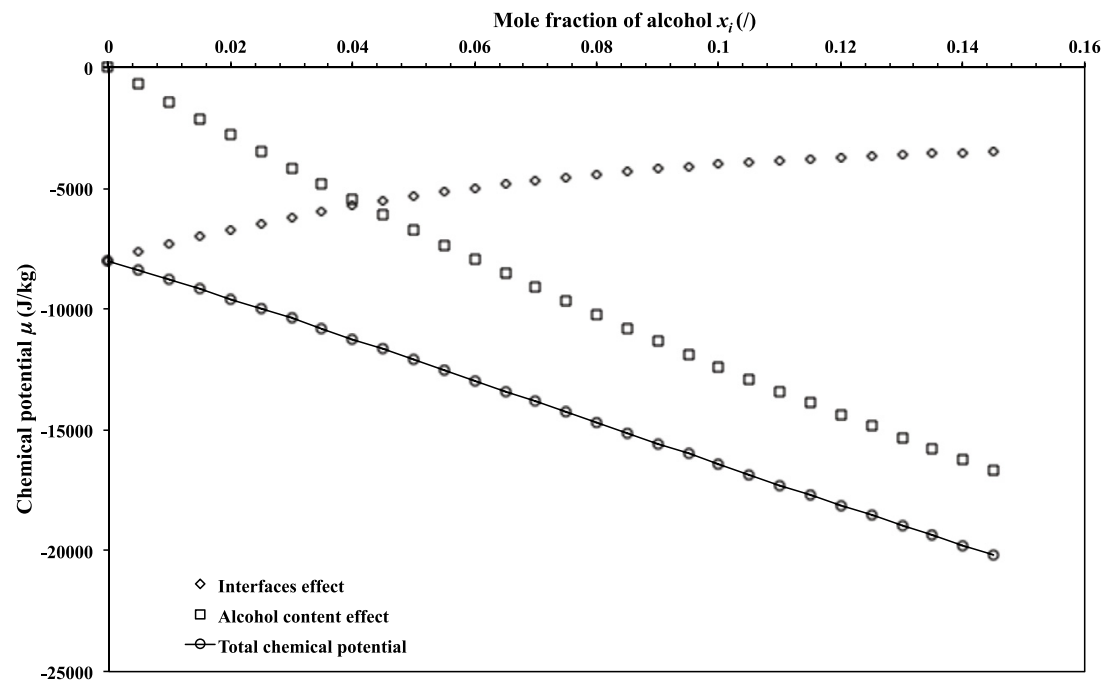

Fig. 6. Variation of the chemical potential of water in relation to $x_{i}$ for $w_{w}^{0}=6 \%$; interface effect and alcohol effect.

in the porous medium, the geometry of the fluid phase is independent of the composition of the solution. This hypothesis is based on the fact of the low compressibility of the fluid on one hand, and the capillary pattern on the other: the shape of the liquid-gas interface is independent of the value of the surface tension so that it is uniform throughout the interface. The presented developments are limited to the water content range where the geometry of the gas-liquid interface is mainly controlled by surface tension.

In this context, the expressions of chemical potentials of the two components were established. They show the superposition of two effects: the effect of interfacial forces and the effect of the presence of the other component.

An interaction study of the water-alcohol solution in clayey silty sand soil was then conducted for the alcohol mole fractions between 0 and 0.15 and the water content ranging from $5 \%$ to $15 \%$. In this range, it was found that the chemical potential of water was affected by the capillary effects below a water content of $10 \%$. Above that, the chemical potential of water was relatively unaffected by the capillary effects and its variation as a function of the mole fraction of alcohol has shown little difference from that of the potential of water in a free solution. The chemical potential of the alcohol was little affected by the capillary effects within the range of water content studied. It varied substantially with the molar fraction of alcohol, in accordance with the variation in a free solution. The presented study emphasizes the water component, and a parallel study could be conducted taking other liquids as reference. This study helps to judge the need to introduce a correction to the values of chemical potential in the case of modelling chemical phenomena, adsorption or phase change in porous media.

The experimental information necessary to consider other solutions includes the equilibrium vapour pressures of the components of the solution in the free state, and the change in surface tension with the molar fraction of 


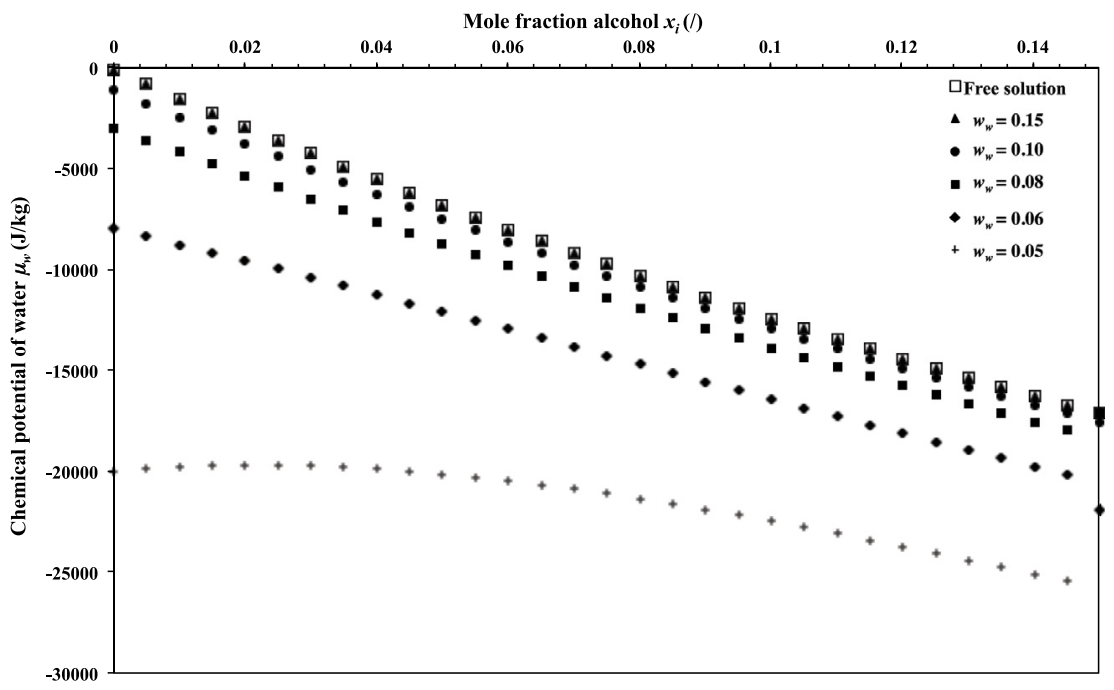

Fig. 7. Change in the chemical potential of water as a function of $x_{i}$ for various water contents.

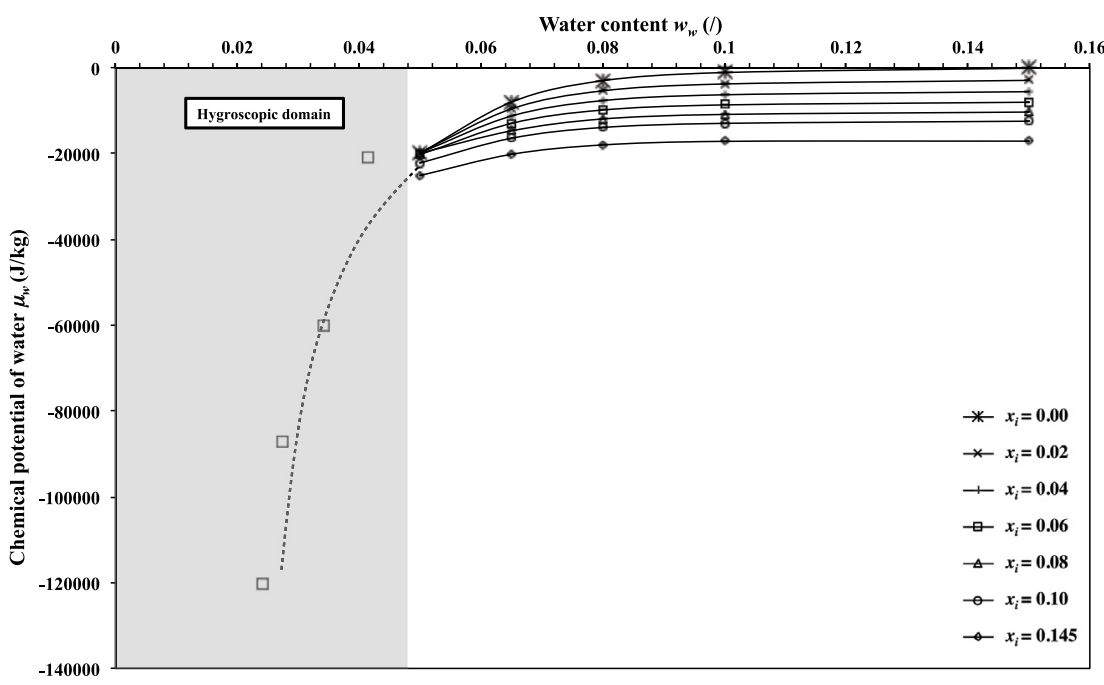

Fig. 8. Variation of the chemical potential of water as a function of the water content for various values of $x_{i}$.

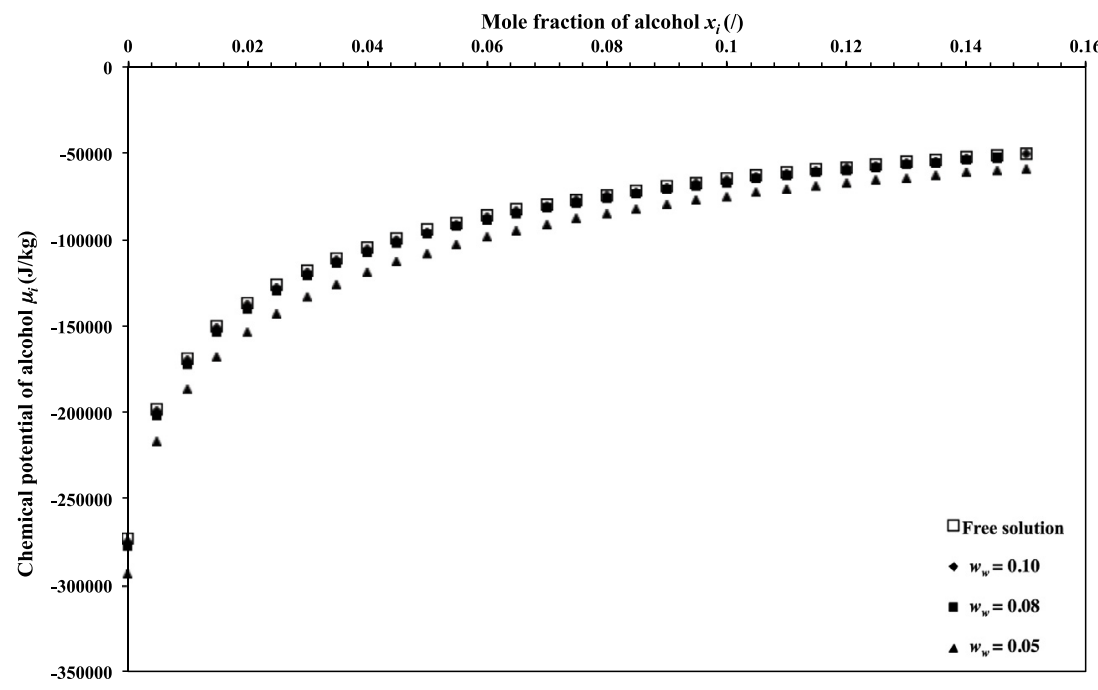

Fig. 9. Variation of the chemical potential of alcohol as a function of $x_{i}$ for various water contents. 
one of the components. For the consideration of any other porous medium, one would obviously have to establish relationship between the water content and chemical potential for the components of the solution in pure form.

\section{References}

[1] Zhao L, Zytner RG. Estimation of SVE closure time. J Hazard Mater. 2008; 153:575-581.

[2] Poulsen TG, Massmann P, Moldrup JW. Effects of vapor extraction on contaminant flux to atmosphere and ground water.J Environ Eng. 1996:122(8):700-706.

[3] Rathfelder KM, Lang JR, Abriola LM. A numerical model (MISER) for the simulation of coupled physical, chemical, and biological processes in soil vapor extraction and bioventing systems. J Contam Hydrol. 2000;43:239-270.

[4] Naon B, Bénet J-C, Cousin B, Cherblanc F, Ali Chammari A. Evaporation of a volatile organic compound in a hygroscopic soil: influence of the airflow and its VOC vapour saturation. Eur J Environ Civil Eng. 2013;17(1):46-63.

[5] Lozano A-L, Cherblanc F, Cousin B, Bénet J-C. Experimental study and modelling of the water phase change kinetics in soils. Eur J Soil Sci. 2008;59(5):939-949.

[6] Bénet J-C, Lozano A-L, Cherblanc F, Cousin B. Phase change of water in a hygroscopic porous medium. Phenomenological relation and experimental analysis for water in soil. J Non-Equilib Thermodyn. 2009;34(2):133-153.
[7] Anoua M, Ramirez-Martinez A, Cherblanc F, Bénet J-C. The use of chemical potential to describe water transfer in complex media with strong solid-liquid bonding. Trans Porous Media. 2014;102:111-122.

[8] Guggenheim EA. Thermodynamique. Paris: Dunod; 1965.

[9] Job G, Hermann F. Chemical potential-a quantity in search of recognition. Eur J Phys. 2006;27:353-371.

[10] Bénet J-C, Ramirez-Martinez A, Ouedraogo F, Cherblanc F. Measurement of the chemical potential of a liquid in porous media. J Porous Media. 2012;15(11):1019-1029.

[11] Callen HB. Thermodynamics and An Introduction to Thermostatistics. 2nd ed. John Wiley and Sons; 1985.

[12] Gagneux G, Millet O. Analytic calculation of capillary bridge properties deduced as an inverse problem from experimental data. Trans Porous Media. 2014;105:117-139.

[13] Salager S, El Youssoufi MS, Saix C. Effect of temperature on water retention phenomena in deformable soils: theoretical and experimental aspects. Eur J Soil Sci. 2010;61:97-107.

[14] Leong EC, Rahardjo H. Permeability functions for UnsatSoils. J Geotech Geoenviron. 1997; 123(12):1118-1126.

[15] Baker R, Frydman S. Unsaturated soil mechanics critical review of physical foundations. Eng Geol. 2009;106:26-39.

[16] Jamin F. Contribution à l'étude du transport de matière et de la rhéologie dans les sols non saturés à différentes températures [Ph.D. thesis], Université Montpellier 2; 2003. 